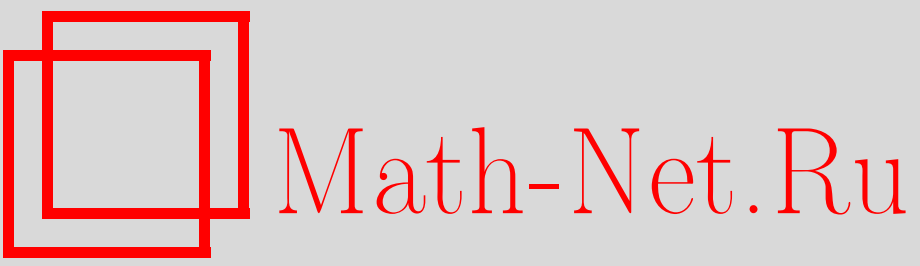

Д. П. Новиков, Р. К. Романовский, Об одном методе построения алгеброгеометрических решений уравнения нулевой кривизны, ТМФ, 1997, том 110, номер 1, 61-72

DOI: https://doi.org/10.4213/tmf952

Использование Общероссийского математического портала Math-Net.Ru подразумевает, что вы прочитали и согласны с пользовательским соглашением

http: //www . mathnet.ru/rus/agreement

Параметры загрузки :

IP : 54.210 .77 .194

26 апреля 2023 г., 17:44:22 


\section{ОБ ОДНОМ МЕТОДЕ ПОСТРОЕНИЯ АЛГЕБРО-ГЕОМЕТРИЧЕСКИХ РЕШЕНИЙ УРАВНЕНИЯ НУЛЕВОЙ КРИВИЗНЫ}

Предлагается конструкция, приводяшая вычисление алгебро-геометрических решений уравнения нулевой кривизны для матриц второго порядка при специальных условиях, выполняемых в ряде приложений, к решению задачи обращения Якоби на гиперэллиптической римановой поверхности и уравнения Риккати. Получено приложение к системе уравнений главного кирального поля.

\section{ВВЕДЕНИЕ}

Будем кратко говорить, что матрица-функция $A(x, y, \lambda),(x, y) \in \mathbb{R}^{2}, \lambda \in \mathbb{C}$, принадлежит классу $Q_{0}$, если она рациональна по $\lambda$ с полюсами, не зависящими от $x, y$. Алгебро-геометрическое решение $(U, V)$ уравнения нулевой кривизны

$$
U_{y}-V_{x}+[U, V]=0
$$

в классе матриц из $Q_{0}$ выделяются требованием (см. [1]) существования матрицы $W=$ $W(U, V) \in Q_{0}$ такой, что

$$
W_{x}=[U, W], \quad W_{y}=[V, W]
$$

(равенства (1), (2) должны выполняться для всех $\lambda$ ). В работе рассматривается система уравнений (1)-(2) для тройки матриц $(U, V, W)$ второго порядка, где матрица $U$ имеет специальный вид: $\mathrm{u}_{i j}(x, y, \lambda)$ - многочлены по $\lambda$ и

$$
\begin{gathered}
\operatorname{deg} \mathrm{u}_{i i} \leqslant 1, \quad \mathrm{u}_{12}=\mathrm{u}_{12}(x, y) \neq 0, \\
\max \left\{\operatorname{deg} \mathrm{u}_{i i}, \operatorname{deg} \operatorname{det} U\right\}=1 \text { или } 2 .
\end{gathered}
$$

Условия (3) выполняются в основных приложениях - см. в $[1,2]$ примеры представлений вида (1) нелинейных уравнений, где $U$ имеет вид (3) либо приводится к (3) преобразованиями вида $(U, V) \rightarrow T(\lambda)(U, V) T^{-1}(\lambda),\left(\lambda-\lambda_{0}\right)^{-1} \rightarrow \lambda$ с рациональной матрицей $T(\lambda)$. Далее без ограничения общности предполагается

$$
\operatorname{Sp} U=\operatorname{Sp} V=\operatorname{Sp} W=0
$$

* Омский государственный технический университет 
(равенства (1)-(3) сохраняются при замене $U \rightarrow U-0.5 \mathrm{Sp} U I$ и таких же заменах для $V, W$; здесь $I$ - единичная матрица). Предлагается подход к построению троек $(U, V, W)$ из $Q_{0}$, удовлетворяюших (1)-(4) (при этом полюсы $V$ заранее не фиксируются), состоящий кратко в следующем.

1. Вначале рассматривается система (1)-(2) для тройки матриц частного вида

$$
\begin{gathered}
U_{0}=\left(\begin{array}{ll}
0 & 1 \\
u & 0
\end{array}\right), \quad V_{0}=\left(\begin{array}{cc}
-0.5 v_{x} & v \\
u v-0.5 v_{x x} & 0.5 v_{x}
\end{array}\right), \\
W_{0}=\left(\begin{array}{cc}
-0.5 w_{x} & w \\
u w-0.5 w_{x x} & 0.5 w_{x}
\end{array}\right) ; \\
u=u_{0}(x, y) \lambda^{2}+u_{1}(x, y) \lambda+u_{2}(x, y), \\
\left|u_{0}\right|+\left|u_{1}\right| \neq 0, \quad w=\prod_{i=1}^{g}\left(\lambda-\gamma_{i}(x, y)\right) \\
v=\sum_{j=0}^{N} v_{j}, \quad v_{0}=\sum_{i=0}^{n} \alpha_{i}(x, y) \lambda^{n-i} \\
v_{j}=\sum_{i=1}^{n_{j}} \beta_{i j}(x, y)\left(\lambda-\lambda_{j}\right)^{-i}, \quad j=\overline{1, N}
\end{gathered}
$$

порядки полюсов $g, n, n_{j}$ и полюсы $\lambda_{j}$ произвольно фиксированы. Показано, что вычисление функций $\gamma_{i}$ приводится к решению задачи обрашения Якоби на кривой $\mu^{2}=\Delta(\lambda)$, где $\Delta(\lambda)$ - многочлен степени $2 g+\operatorname{deg} u$, решаемой в тета-функциях [3]. Коэффициенты $u_{i}$ вычисляются по $w, \Delta$ из системы линейных алгебраических уравнений. Основной результат в этом пункте - представление функции $v$ через нули многочлена $w$ (формулы (22), (25)). Получаемое множество решений (5) системы (1)-(2) при фиксированных полюсах $(6),(7)$ и многочлене $\Delta(\lambda)$ зависит от произвольной гладкой вектор-функции $c(y)$ размера $n+1+\sum_{j} n_{j}$ и произвольного вектора размера $g$ и содержит все решения вида (5)-(7).

2. Рассматривается система (1)-(2) при условиях $(3),(4)$ и дополнительном условии на матрищу $W$ : элемент $\mathrm{w}_{12}$ ишется в виде

$$
\mathrm{w}_{12}=\mathrm{u}_{12}(x, y) w(x, y, \lambda)
$$

где $w$ - многочлен вида (6) произвольно фиксированной степени $g$. Показано, что каждое решение $(U, V, W)$ из $Q_{0}$ переводится в решение вида $(5)-(7)$ преобразованием

$$
U_{0}=G_{x} G^{-1}+G U G^{-1}, \quad V_{0}=G_{y} G^{-1}+G V G^{-1}
$$

где $G$ строится по матрице $U$ (формула (32)), $G, G^{-1} \in Q_{0}$, при этом $v=\mathrm{v}_{12} \mathrm{u}_{12}^{-1}$ и коэффициенты многочленов

$$
\mathrm{u}_{11}=a(x, y) \lambda+b(x, y), \quad \operatorname{det} U=A(x, y) \lambda^{2}+B(x, y) \lambda+C(x, y)
$$


связаны с коэффициентами многочлена $u$ в (6) равенствами

$$
\begin{gathered}
A=-u_{0}, \quad a_{x}-a z=u_{1}+B, \\
0.5 z_{x}+b z-0.25 z^{2}=f, \quad z=\mathrm{u}_{12 x} \mathrm{u}_{12}^{-1}, \quad f=-u_{2}+b_{x}-C .
\end{gathered}
$$

3. Каждому решению (5)-(7) системы (1)-(2), найденному указанным в пункте 1 способом, отвечает в силу равенств (9)-(11) семейство решений $(U, V, W)$ из $Q_{0}$ системы (1)-(4), зависящее от вектор-функции $F(x, y)=(a, b, A, B, C, z)$, удовлетворяюшей (11). Очевидно, множество допустимых $F$ может быть параметризовано тройкой $(b, B, C)$ произвольных гладких функций от $x, y$ и тройкой произвольных гладких функций от $y$ - “постоянных" интегрирования, сводяшегося к решению уравнения Риккати. Строяшееся в итоге семейство решений $(U, V)$ уравнения $(1)$ зависит от набора данных $g, \Delta(\lambda), n, n_{j}, \lambda_{j}, F, c(y)$, указанных выше.

4. Изложенная конструкция дает подход к построению алгебро-геометрических решений нелинейных уравнений с частными производными, представимых в виде (1), (3). В этом случае полюсы $V$ заданы и между элементами $U, V$ имеются связи помимо (1), (3). Задаваясь порождающей матрицей $W$ вида $(4),(8)$, переходя к тройке (5)-(7) по формулам (9), решая систему (1)-(2) для этой тройки при фиксированных $g, \Delta(\lambda)$ (в этой ситуации числа $n, n_{j}, \lambda_{j}$ заданы, см. п. 2$)$ и возврашаясь назад (п. 3 ), нужно использовать произвол в выборе $F$ для удовлетворения дополнительных соотношений. В качестве примера в работе построен класс алгебро-геометрических решений системы уравнений главного кирального поля.

Далее система (1)-(4), (8) для матриц (5)-(7) называется канонической в классе таких систем.

\section{1. ОПИСАНИЕ РЕШЕНИЙ КАНОНИЧЕСКОЙ СИСТЕМЫ}

Далее, говоря о равенствах, содержащих параметр $\lambda$, будем опускать слова “при всех $\lambda "$. Зафиксируем произвольно числа $g, n, n_{j}, \lambda_{j}$ в $(6),(7)$.

ЛЕмма 1. Следующие утвержсдения равносильны:

(i) Матрицы (5)-(7) удовлетворяют равенствам (1), (2) на открытом множестве $D \subset \mathbb{R}^{2}$.

(ii) На D справедливы равенства

$$
\begin{gathered}
-0.5 w w_{x x}+0.25 w_{x}^{2}+u w^{2}=\Delta(\lambda), \\
w_{y}=v w_{x}-w v_{x}
\end{gathered}
$$

где $\Delta(\lambda)$ - многочлен степени $2 g+\operatorname{deg} u$.

ДокАЗАТЕЛЬСтво. Для обоснования (ii) $\rightarrow($ i) нужно вывести из (12), (13) равенства

$$
\begin{gathered}
0.5 w_{x x x}-2 u w_{x}-u_{x} w=0, \quad w_{x y}=w_{x x} v-w v_{x x} \\
0.5\left(w_{x x y}+v_{x} w_{x x}-v_{x x} w_{x}\right)-2 u w_{y}-u_{y} w=0 \\
u_{y}-v u_{x}-2 u v_{x}+0.5 v_{x x x}=0
\end{gathered}
$$

3 Теоретическая и математическая физика, т. 110, № 1, 1997 г. 
(система (1)-(2) для матриц (5)-(7) равносильна системе (13)-(14)). Первые два получаются из $(12),(13)$ дифференцированием по $x$, третье - дифференцированием (12) по $y$ с учетом (13), четвертое получается из первых трех и (13) исключением функции $w$ и ее производных (при этом второе равенство (14) нужно предварительно продифференцировать по $x$ ). Обратно, пусть верны (13), (14). Умножая первое равенство (14) на $w$, получим $L_{x}=0$, где $L(x, y, \lambda)$ - левая часть (12). Далее, умножая третье равенство (14) на $w$ и подставляя в него выражения для $w v_{x}$ и $w v_{x x}$ из (13) и второго равенства (14), получим $L_{y}=0$. Отсюда следует $L=\Delta, \Delta=\Delta(\lambda)$; так как $L$ - многочлен степени $2 g+\operatorname{deg} u$ по $\lambda$, то же верно для $\Delta(\lambda)$.

Лемма 1 приводит построение решений канонической системы к решению системы (12)-(13) при произвольно фиксированном многочлене

$$
\Delta(\lambda)=\sum_{i=0}^{2 g+2} \Delta_{i} \lambda^{2 g+2-i}, \quad\left|\Delta_{0}\right|+\left|\Delta_{1}\right| \neq 0
$$

Предполагая, что $\Delta(\lambda)$ не имеет кратных корней, построим компактную риманову поверхность $\Gamma$ с уравнением $\mu^{2}=\Delta(\lambda)$ и рассмотрим систему уравнений на $\Gamma$

$$
\sum_{i=1}^{g} \int_{\left(\infty, \sigma_{i}\right)}^{\left(\gamma_{i}, \sigma_{i}\right)} \frac{\lambda^{k-1} d \lambda}{2 \sqrt{\Delta(\lambda)}}=\delta_{k g} x+\xi_{k}(y), \quad k=\overline{1, g}
$$

где при каждом $i$ путь интегрирования во всех уравнениях один и тот же, $\sigma_{i}= \pm$ (в случае $\Delta_{0}=0$ имеем $\left.(\infty,+)=(\infty,-)\right), \delta_{i j}$ - символ Кронекера, $\xi_{k}(y)$ - произвольные функции. После фиксирования базиса циклов система (15) приводится к задаче обрашения Якоби на $\Gamma$ (см. ниже раздел 3 ). Будем называть открытое множество $D \subset \mathbb{R}^{2}$ множеством общего положения относительно многочлена $w$, если

$$
\gamma_{i} \neq \gamma_{j} \quad(i \neq j), \quad \Delta\left(\gamma_{i}\right) \neq 0 \text { при } \quad(x, y) \in D .
$$

ЛЕмма 2. При условиях общего положения (16) следующие утверждения равносильнь:

(i) Функиии (6) удовлетворяют уравнению (12) на D.

(ii) На D выполняются равенства (15) при некоторых $\sigma_{i}, \xi_{k} u$

$$
u_{0}=\Delta_{0}, \quad u_{1}=\Delta_{1}-2 \Delta_{0} w_{1}, \quad u_{2}=\Delta_{2}-2 \Delta_{1} w_{1}+\Delta_{0}\left(3 w_{1}^{2}-2 w_{2}\right),
$$

где $w_{i}$ - коәффициент при $\lambda^{g-i}$ многочлена $w$.

ДокАЗАТЕЛЬСТво. При условиях (16) первая часть утверждения (ii) равносильна утверждению о справедливости равенств

$$
0.25 \Delta^{-1}\left(\gamma_{i}\right) \gamma_{i x}^{2}=\prod_{j \neq i}\left(\gamma_{i}-\gamma_{j}\right)^{-2}, \quad i=\overline{1, g}
$$


(см. $[3,4])$. Равенства (18) получаются из (12) подстановкой $\lambda=\gamma_{j}$. Равенства $(17)$ означают совпадение трех старших коэффициентов в левой и правой частях (12). Тем самым (i) $\rightarrow$ (ii). Далее, равенства (18) означают выполнение соотношений

$$
(L-\Delta)_{\lambda=\gamma_{i}}=0, \quad i=\overline{1, g},
$$

для левой части $L$ равенства (12). Дифференцируя (19) по $x$ и пользуясь тем, что $L_{x}\left(x, y, \gamma_{i}\right)=0$, получим

$$
\left.\frac{\partial}{\partial \lambda}(L-\Delta)\right|_{\lambda=\gamma_{i}}=0, \quad i=\overline{1, g} .
$$

В силу выбора функций $u_{i}$ в каждой точке $(x, y) \in D$ три старших коэфффициента многочлена $L-\Delta$ равны нулю. Равенства (19), (20) представляют собой систему $2 g$ линейных однородных уравнений для остальных $2 g$ коэффициентов с определителем, не равным нулю. Отсюда следует $L=\Delta$ на $D$, т.е. (ii) $\rightarrow(\mathrm{i})$.

Пусть: 1) $\left(\gamma_{1}, \ldots, \gamma_{g}\right)$ - решение системы (15) при некоторых гладких $\xi_{k}(y): \gamma_{i}=$ $f_{i}(x, \xi(y)), \xi=\left(\xi_{1}, \ldots, \xi_{g}\right), i=\overline{1, g}$;

2) $w$ - многочлен (6) с нулями $\gamma_{i}=f_{i}$;

$3)$ функции $\varphi_{m}(x, y, \lambda), \varphi_{j m}(x, y, \lambda)$ даются формулами

$$
\begin{gathered}
\varphi_{m}= \begin{cases}1, & m=0, \\
\lambda^{m}+\sum_{i=1}^{m} w_{i} \lambda^{m-i}, & 1 \leqslant m \leqslant g, \\
\lambda^{m-g} w, & m>g,\end{cases} \\
\varphi_{j m}= \begin{cases}\sum_{i=g-m+1}^{g} w_{i j}\left(\lambda-\lambda_{j}\right)^{g-i-m}, & 1 \leqslant m \leqslant g, \\
\left(\lambda-\lambda_{j}\right)^{-m} w, & m>g,\end{cases}
\end{gathered}
$$

где $\lambda_{j}$ - полюсы (7), $w_{i j}$ - коэффициент при $\left(\lambda-\lambda_{j}\right)^{g-i}$ в разложении $w$ по степеням $\lambda-\lambda_{j}$

4) функция $\varphi=\varphi(x, \xi(y), c(y), \lambda)$ дается формулой

$$
\varphi=\sum_{m=0}^{n} c_{m}(y) \varphi_{m}+\sum_{j=1}^{N} \sum_{m=1}^{n_{j}} c_{j m}(y) \varphi_{j m},
$$

где $n, n_{j}$ - порядки полюсов $(7), c(y)=\left(c_{m}, c_{j m}\right)$ - набор $n+1+\sum_{j} n_{j}$ произвольных гладких функций $\mathbb{R} \rightarrow \mathbb{C}$.

Теорема 1. Пусть в условиях 1-4 вектор-функиии $\xi(y), c(y)$ связаны соотношениями

$$
\begin{gathered}
\xi_{k y}=\varepsilon_{k} c_{g-k}(y)-\sum_{j=1}^{N} \sum_{m=1}^{n_{j k}} \mathrm{C}_{k-1}^{k-m} \lambda_{j}^{k-m} c_{j m}(y), \quad k=\overline{1, g} \\
\varepsilon_{k}=\left\{\begin{array}{ll}
1, & g-k>n, \\
0, & g-k \leqslant n,
\end{array} n_{j k}=\min \left\{n_{j}, k\right\}\right.
\end{gathered}
$$


и открытое множество $D \subset \mathbb{R}^{2}$ таково, что для функиий $\gamma_{i}=f_{i}$ выполнень условия общего положения (16) и множества $\gamma_{i}(D)$ отделены от полюсов $\lambda_{j}$ :

$$
\min _{i, j} \operatorname{dist}\left\{\gamma_{i}(D), \lambda_{j}\right\}>0
$$

Тогда функиии

$$
v(x, y, \lambda)=\varphi, \quad w(x, y, \lambda)=\prod_{i=1}^{g}\left(\lambda-f_{i}\right)
$$

удовлетворяют уравнению (13) на D. Решения (25), зависящие от произвольной гладкой вектор-функции с (у) и постоянных интегрирования в (23), исчерпывают все решения вида (6), (7) уравнения (13).

ДокАЗАТЕЛЬСтво. 1. Уравнение (13) для функций $v, w$ вида (6), (7) равносильно системе

$$
\begin{gathered}
\gamma_{i y}=\gamma_{i x} v\left(x, y, \gamma_{i}\right), \quad i=\overline{1, g} \\
L_{j}=\sum_{i=1}^{g} \gamma_{i x} \frac{v_{j}(x, y, \lambda)-v_{j}\left(x, y, \gamma_{i}\right)}{\lambda-\gamma_{i}}+v_{j x}(x, y, \lambda)=0, \quad j=\overline{0, N} .
\end{gathered}
$$

В самом деле, из (13) при $\lambda=\gamma_{i}$ следует (26). Далее, умножая обе части (13) на $w^{-1}$ и учитывая (26), получим $\sum_{j} L_{j}=0$. Так как при $(x, y) \in D$ рациональная дробь $L_{j}(\lambda)$ имеет в силу (25) единственный полюс $\lambda_{j}$ (здесь $\lambda_{0}=\infty$ ), то отсюда следует $L_{j}=0$ при каждом $j$. Обратно, складывая равенства (27) и учитывая (26), получим (13).

2. Покажем, что равенства (26) для функций (25) равносильны условиям (23). Дифференцируя (15) по $y$, получим систему $g$ линейных уравнений относительно $\gamma_{1 y}, \ldots, \gamma_{g y}$ с определителем, не равным нулю, откуда следует, что равенства (26) для функций (25) равносильны равенствам

$$
\xi_{k y}=\sum_{i=1}^{g} \frac{\gamma_{i}^{k-1} \gamma_{i x} \varphi\left(x, \xi, c, \gamma_{i}\right)}{2 \sqrt{\Delta\left(\gamma_{i}\right)}}, \quad k=\overline{1, g}
$$

вытекаюшим из этой системы с учетом (26) и $v=\varphi$. Используя равенства (19), (22), нетрудно привести (28) к виду

$$
\begin{gathered}
\xi_{k y}=\sum_{m=0}^{n} c_{m} r_{k m}+\sum_{j=1}^{N} \sum_{m=1}^{n_{j}} c_{j m} r_{j k m}, \quad k=\overline{1, g} \\
r_{k m}=-\operatorname{res}_{\infty} \lambda^{k-1} \varphi_{m} w^{-1}, \quad r_{j k m}=-\operatorname{res}_{\lambda_{j}} \lambda^{k-1} \varphi_{j m} w^{-1} .
\end{gathered}
$$

Вычисления дают

$$
r_{k m}=\delta_{m, g-k}, \quad r_{j k m}= \begin{cases}-\mathrm{C}_{k-1}^{k-m} \lambda_{j}^{k-m}, & m \leqslant k, \\ 0, & m>k .\end{cases}
$$


Отсюда следует требуемое.

В силу пунктов 1,2 и формул (7), (22) для $v, w$ для доказательства теоремы достаточно показать справедливость в $D$ при произвольных $c_{m}(y), c_{j m}(y)$ соотношений

$$
\begin{aligned}
L_{0} & =0 \Leftrightarrow v_{0}=\sum_{m=0}^{n} c_{m}(y) \varphi_{m}, \\
L_{j} & =0 \Leftrightarrow v_{j}=\sum_{m=1}^{n_{j}} c_{j m}(y) \varphi_{j m}, \quad j=\overline{1, N} .
\end{aligned}
$$

3. Покажем справедливость (29). При $n=0$ оно очевидно. Пусть $n \geqslant 1, K-$ компакт в $D$, число $R>0$ таково, что множества $\gamma_{i}(K)$ лежат в круге $|\lambda|<R$. Умножая $(27)$ на $\lambda^{k-1} w^{-1}$ и интегрируя по окружности $|\lambda|=R$, получим

$$
\mathcal{L}_{k} v_{0}=\frac{\partial}{\partial x} \int_{|\lambda|=R} \lambda^{k-1} \frac{v_{0}}{w} d \lambda=0, \quad g-n \leqslant k \leqslant g
$$

(при $k<g-n(31)$ тривиально). Уравнение $L_{0}=0$ и система (31) содержат одну и ту же информацию о векторе $\alpha(x, y)=\left(\alpha_{0}, \ldots, \alpha_{n}\right)$ коэффициентов многочлена $v_{0}$ на компакте $K$. В самом деле, равенства $L_{0}=0$ и (31), как нетрудно убедиться, равносильны линейным системам соответственно вида $\alpha_{x}=B \alpha, A \alpha_{x}=B_{1} \alpha$ для вектора $\alpha$ на множестве $K_{x}$ - проекции $K$ на ось $x$, где $A$-нижняя треугольная матрица с элементами $a_{i i}=1$, вследствие чего она обратима. Отсюда вытекает, что при каждом $y \in K_{y}$ линейные пространства $H_{1}(y), H_{2}(y)$ решений этих систем имеют одинаковую размерность $n+1$. Так как $H_{1} \subset H_{2}$ (система (31) выведена из равенства $L_{0}=0$ ), то $H_{1}=H_{2}$. Далее, простые вычисления с учетом $(21)$ дают $\mathcal{L}_{k} \varphi_{m}=0, m \geqslant 0$, где $\varphi_{m}$ - функции $(21), \mathcal{L}_{k}$ - операторы (31). Это означает, что векторы коэффициентов многочленов $\varphi_{0}, \ldots, \varphi_{n}$ в $(29)$, дополненные нулями до размера $n+1$, содержатся в $H_{2}$, а следовательно, они содержатся в $H_{1}$. Так как они линейно независимы, то образуют базис в $H_{1}$. Тем самым доказана справедливость $(29)$ на любом компакте $K \subset D$, а значит, оно верно в $D$.

4. В силу условия (24) существует набор попарно непересекающихся кругов $\left|\lambda-\lambda_{j}\right| \leqslant R_{j}, j=\overline{1, N}$, такой, что множества $\gamma_{i}(D)$ имеют пустое пересечение с каждым из них. С помошью рассуждений, аналогичных проведенным в пункте 3 , получим, что равенство $L_{j}=0$ при $j \neq 0$ равносильно в $D$ системе уравнений

$$
\frac{\partial}{\partial x} \int_{\left|\lambda-\lambda_{j}\right|=R_{j}}\left(\lambda-\lambda_{j}\right)^{k-1} \frac{v_{j}}{w} d \lambda=0, \quad 1 \leqslant k \leqslant n_{j},
$$

и верно соотношение (30); при этом сушественно, что в формуле (21) для $\varphi_{j m}$ "старшие" коэффициенты $w_{g j}=\prod_{i=1}^{g}\left(\lambda_{j}-\gamma_{i}\right) \neq 0$ в $D$. Теорема доказана.

Из лемм 1,2 и теоремы 1 вытекает

Теорема 2. В условиях теоремь 1 матриць (5), әде функиии $и, v, w$ даются формулами (6), (7), (22), (25), удовлетворяют системе (1)-(2). Получаемые таким путем решения исчерпьвают все решения вида (5)-(7). 


\section{2. СЕМЕЙСТВО РЕШЕНИЙ СИСТЕМЫ (1)-(4)}

Построим по матрице $U$ вида (3) матрицу

$$
G=\mathrm{u}_{12}^{-0.5}\left(\begin{array}{cc}
1 & 0 \\
\mathrm{u}_{11}-0.5 z & \mathrm{u}_{12}
\end{array}\right), \quad z=\frac{\mathrm{u}_{12 x}}{\mathrm{u}_{12}} .
$$

Лемма 3. Преобразование (9) с матрицей (32) переводит решение $(U, V, W)$ класса $Q_{0}$ системь (1)-(4), где W удовлетворяет дополнительному условию (8), в решение вида (5)-(7), где

$$
\begin{gathered}
u=-A \lambda^{2}+\left(a_{x}-a z-B\right) \lambda-\left(0.5 z_{x}+b z-0.25 z^{2}-b_{x}+C\right), \\
v=\mathrm{v}_{12} \mathrm{u}_{12}^{-1}
\end{gathered}
$$

$a, b, A, B, C, z-$ функиии (10), (32).

Доказательство. Преобразование (9) сохраняет равенства (1), (2). Формулы (5), (33) для $U_{0}, v$ получаются прямым вычислением, формулы для остальных элементов матрищ $V_{0}, W_{0}$ следуют из $(8)$ и равенств $(1),(2)$ для матрищ $(5)$.

Теорема 3. Пусть $\left(U_{0}, V_{0}, W_{0}\right)$-решение (5)-(7) системы (1)-(2), построенное указанным в теореме 2 способом, и функиии $a, b, A, B, C, z$ переменных $x, y$ удовлетворяют равенствам (11), где и

1. Тройка матрии $(U, V, W)$, где әлементы $U$ вычислены из (10), (32) с учетом $\operatorname{Sp} U=0$ и $V, W$ вычислень из (9) с матрицей (32), - решение класса $Q_{0}$ системыз (1)-(4), при этом $\mathrm{v}_{12}=v \mathrm{u}_{12}, \mathrm{w}_{12}=w \mathrm{u}_{12}$.

2. Получаемое таким путем множсество алгебро-геометрических решений $(U, V)$ уравнения нулевой кривизны исчерпьвает все решения вида (3)-(4), для которых порождающая матрица $W$ удовлетворяет условию (8).

ДокаЗАтельство. Равенства (11) равносильны формуле (33) для многочлена $u$ в (5). При указанном способе построения тройка $(U, V, W)$ связана с тройкой $\left(U_{0}, V_{0}, W_{0}\right)$ с учетом леммы 3 равенствами (5), где $G$ - матрица (32), вычисленная по $U$. Отсюда следует первое утверждение теоремы. Второе утверждение следует из теоремы 2 и леммы 3.

Из теоремы 3 следуют утверждения, сформулированные в п. 3 введения.

\section{3. КЛАСС АЛГЕБРО-ГЕОМЕТРИЧЕСКИХ РЕШЕНИЙ СИСТЕМЫ УРАВНЕНИЙ ГЛАВНОГО КИРАЛЬНОГО ПОЛЯ}

Уравнение нулевой кривизны (1) для матриц

$$
U=\left(\begin{array}{cc}
U_{11} \lambda & U_{12} \\
U_{21} \lambda^{2} & -U_{11} \lambda
\end{array}\right), \quad V=\left(\begin{array}{cc}
V_{11} \lambda & V_{12} \\
V_{21} \lambda^{2} & -V_{11} \lambda
\end{array}\right)(\lambda-1)^{-1}
$$

равносильно, как нетрудно убедиться, системе уравнений главного кирального поля $[1,5]$

$$
\mathrm{u}_{y}+[\mathrm{u}, \mathrm{v}]=0, \quad \mathrm{v}_{x}-[\mathrm{u}, \mathrm{v}]=0
$$


для матриц $\mathrm{u}=\left\|U_{i j}(x, y)\right\|, \mathrm{v}=\left\|V_{i j}(x, y)\right\|$ (в (35) можно без ограничения общности считать $\mathrm{Sp} \mathrm{u}=\mathrm{Spv}=0$ ). Ниже на основе изложенных приемов строится класс алгебро-геометрических решений уравнения (1) для пары (34) с порождающей матрицей $W$, удовлетворяюшей условиям (4) и (8), при

$$
w=\left(\lambda-\gamma_{1}\right)\left(\lambda-\gamma_{2}\right), \quad \Delta(\lambda)=\lambda \prod_{i=1}^{4}\left(\lambda-E_{i}\right),
$$

$E_{i} \neq 0, E_{i} \neq E_{j}$ при $i \neq j$. При решении задачи обрашения Якоби (36) была использована формула (2.4.29) из работы [3] для сумм $\sum_{i} \gamma_{i}^{s}$. Специальный вид матришы $U$ и многочлена $\Delta(\lambda)(\lambda=0$ - корень) позволил найти точное частное решение уравнения Риккати (11). На последнем этапе при вычислении матрицы $V$ подбором постоянных интегрирования отсекались “лишшие" слагаемые; здесь также использовалось решение задачи обрашения Якоби, в том числе формула (39).

1. Пусть $\Gamma$ - компактная риманова поверхность $\mu^{2}=\Delta(\lambda)$. Построим по стандартной методике [3] канонический базис циклов $a_{1}, a_{2}, b_{1}, b_{2}$ на $\Gamma$ и канонический базис голоморфных дифференциалов

$$
\omega_{k}=\left(\alpha_{k 1} \lambda+\alpha_{k 2}\right) \sqrt{\Delta^{-1}(\lambda)} d \lambda, \quad k=1,2,
$$

и пусть $\theta\left(z_{1}, z_{2}\right)$ - тэта-функция поверхности Г с матрицей Римана, вычисленной в этом базисе [6]. Умножая столбец равенств (15) при $g=2$ слева на матрицу $2\left\|\alpha_{i j}\right\|\left(\begin{array}{ll}0 & 1 \\ 1 & 0\end{array}\right)$, получим систему

$$
\begin{gathered}
\int_{\infty}^{\left(\gamma_{1}, \sigma_{1}\right)} \omega_{k}+\int_{\infty}^{\left(\gamma_{2}, \sigma_{2}\right)} \omega_{k}=\zeta_{k}, \\
\zeta_{k}=2\left[\alpha_{k 1}\left(x+\xi_{1}(y)\right)+\alpha_{k 2} \xi_{2}(y)\right], \quad k=1,2 .
\end{gathered}
$$

Вычисления по формуле (2.4.29) из [3] (аналогичные проведенным там) с учетом формул для $\zeta_{k}$ дают

$$
\begin{gathered}
w_{1}=-\left(\gamma_{1}+\gamma_{2}\right)=\frac{\partial^{2}}{\partial x^{2}} \ln \theta(\zeta)-c_{1}, \\
\gamma_{1}^{2}+\gamma_{2}^{2}=\frac{1}{6}\left(-\frac{\partial^{4}}{\partial x^{4}}-8 \frac{\partial^{2}}{\partial x \partial t}+4 \Delta_{2} \frac{\partial^{2}}{\partial x^{2}}\right) \ln \theta(\zeta)+c_{2}, \\
\gamma_{1}^{3}+\gamma_{2}^{3}=-\frac{1}{120} \eta \ln \theta(\zeta)+c_{3}, \\
\eta=\frac{\partial^{6}}{\partial x^{6}}+40 \frac{\partial^{4}}{\partial x^{3} \partial t}+40 \frac{\partial^{2}}{\partial t^{2}}-20 \Delta_{2} \frac{\partial^{4}}{\partial x^{4}}- \\
-112 \Delta_{2} \frac{\partial^{2}}{\partial x \partial t}+\left(-72 \Delta_{3}+64 \Delta_{2}^{2}\right) \frac{\partial^{2}}{\partial x^{2}} \\
\frac{\partial}{\partial t}=2\left(\alpha_{12} \frac{\partial}{\partial \zeta_{1}}+\alpha_{22} \frac{\partial}{\partial \zeta_{2}}\right), \quad c_{k}=\frac{1}{2 \pi i}\left(\int_{a_{1}} \lambda^{k} \omega_{1}+\int_{a_{2}} \lambda^{k} \omega_{2}\right),
\end{gathered}
$$


$k=1,2,3$, где $\zeta=\left(\zeta_{1}, \zeta_{2}\right), \Delta_{k}-$ коэффициенты $\Delta(\lambda)$ в обозначениях раздела $1 ;$ вектор римановых констант "прячется" в произвольных $\xi_{k}(y)$. Для получения нужного далее представления коэффициента $w_{2}=\gamma_{1} \gamma_{2}$ многочлена $w$ воспользуемся формулой (18). Умножая (18) на $\Delta\left(\gamma_{i}\right)$, дифференцируя по $x$, выражая отсюда $\gamma_{i x x}$ и складывая полученные равенства, после простых вычислений найдем

$$
\left(\gamma_{1}+\gamma_{2}\right)_{x x}-4 \Delta_{2}\left(\gamma_{1}+\gamma_{2}\right)-6\left(\gamma_{1}^{2}+\gamma_{2}^{2}\right)-8 \gamma_{1} \gamma_{2}-2 \Delta_{3}=0
$$

откуда с учетом (37), (38) следует

$$
w_{2}=\gamma_{1} \gamma_{2}=\frac{\partial^{2}}{\partial x \partial t} \ln \theta(\zeta)-c, \quad c=0.5 c_{1} \Delta_{2}+0.75 c_{2}+0.25 \Delta_{3}
$$

2. Выполним переход к соответствующей канонической тройке (5), используя преобразование (9) с матрицей (32). Из (33), (34) следует, что функция (7) имеет здесь вид $v=\beta_{11}(x, y)(\lambda-1)^{-1}$. Применяя теоремы 1,2 , получим

$$
v=c_{11}(y) \frac{w(x, y, 1)}{\lambda-1}=c_{11}(y) \frac{1+w_{1}+w_{2}}{\lambda-1},
$$

где $w_{k}$ - функции $(37),(38), c_{11}(y)$ - произвольная гладкая функция, при этом функции $\xi_{k}(y)$ в (36) удовлетворяют соотношениям

$$
\xi_{1 y}=\xi_{2 y}=-c_{11}(y)
$$

Из (17) с учетом равенств $\Delta_{0}=0, \Delta_{1}=1$ вытекает

$$
u=\lambda+\Delta_{2}-2 w_{1} .
$$

3. В формулах (10), (11) с учетом (34), (44) имеем $a=U_{11}, b=A=B=C=0 \Rightarrow$ $\operatorname{det} U=0$, поэтому

$$
\begin{gathered}
U_{11}^{2}+U_{12} U_{21}=0, \quad U_{11 x}-z U_{11}=1, \quad\left(\ln U_{12}\right)_{x}=z, \\
0.5 z_{x}-0.25 z^{2}=2 w_{1}-\Delta_{2} .
\end{gathered}
$$

Подставляя в (12) $\lambda=0$ и учитывая (44), получим, что функция

$$
z=-\left(\ln w_{2}\right)_{x}
$$

является решением уравнения (46). Из (41), (45), (47) следуют формулы

$$
U_{11}=\left[\frac{\partial}{\partial t} \ln \theta(\zeta)-c x+r_{1}\right] w_{2}^{-1}, \quad U_{12}=r_{2} w_{2}^{-1}, \quad U_{21}=-U_{11}^{2} U_{12}^{-1}
$$

где $r_{k}(y)$ - "постоянные" интегрирования, подлежашие уточнению далее. 
4. Найдем матрицу $V$ из второго равенства (9). Вычисления с учетом формул (18), (33), (42), (44)-(48) дают

$$
\begin{aligned}
V & =\left(V_{1}+V_{2}\right)(\lambda-1)^{-1}, \\
V_{1} & =\left(\begin{array}{cc}
\left(V_{11}+\frac{r_{2}^{\prime}}{r_{2}}\right) \lambda & V_{12} \\
V_{21} \lambda^{2} & -\left(V_{11}+\frac{r_{2}^{\prime}}{r_{2}}\right) \lambda
\end{array}\right), \\
V_{2} & =\left(\begin{array}{cc}
\varepsilon_{11}-\frac{r_{2}^{\prime}}{2 r_{2}} & 0 \\
\left(\varepsilon_{21} \lambda+\varepsilon_{11 x}\right) U_{12}^{-1} & -\varepsilon_{11}+\frac{r_{2}^{\prime}}{2 r_{2}}
\end{array}\right),
\end{aligned}
$$

где

$$
\begin{gathered}
V_{11}=c_{11}(y)\left(1+w_{1}+w_{2}\right) U_{11}-w_{2 y} w_{2}^{-1}, \\
V_{12}=c_{11}(y)\left(1+w_{1}+w_{2}\right) w_{2}^{-1}, \\
V_{21}=-\left[c_{11}(y)\left(1+w_{1}+w_{2}\right) U_{11}^{2}+U_{11 y}\right] U_{12}^{-1} ; \\
\varepsilon_{11}=0.5\left\{w_{2 y} w_{2}^{-1}-c_{11}(y)\left[\left(1+w_{1}+w_{2}\right)_{x}-z\left(1+w_{1}+w_{2}\right)\right]\right\} ; \\
w_{2} \varepsilon_{21}=r_{1}^{\prime}+\frac{\partial^{2}}{\partial y \partial t} \ln \theta(\zeta)+ \\
+c_{11}(y)\left\{w_{2}+L_{1}\left[\gamma_{1}+\gamma_{2}\right]+L_{2}\left[\gamma_{1}^{2}+\gamma_{2}^{2}\right]+L_{3}\left[\gamma_{1}^{3}+\gamma_{2}^{3}\right]\right\} ; \\
L_{1}[\mathrm{u}]=-0.1 \mathrm{u}_{x x x x}+0.35 \Delta_{2} \mathrm{u}_{x x}+\left(0.2 \Delta_{2}^{2}-1.8 \Delta_{3}\right) \mathrm{u}, \\
L_{2}[\mathrm{u}]=0.75 \mathrm{u}_{x x}-2.1 \Delta_{2} \mathrm{u}, \quad L_{3}[\mathrm{u}]=-3 \mathrm{u}+0.1 \Delta_{2} \Delta_{3}-1.2 \Delta_{4}+\Delta_{5} .
\end{gathered}
$$

Из (51) с учетом $(26),(42),(47)$ нетрудно получить $\varepsilon_{11}=0$. Подставляя в (52) функции (37)-(39), (41), после вычислений с учетом вытекающей из (36), (40), (43) формулы

$$
\frac{\partial}{\partial y}=-c_{11}(y)\left(\frac{\partial}{\partial x}+\frac{\partial}{\partial t}\right)
$$

и формулы (41) для постоянной $c$ получим

$$
\begin{gathered}
w_{2} \varepsilon_{21}=r_{1}^{\prime}-\nu c_{11}(y) \\
\nu=\left(0.5 \Delta_{2}-0.2 \Delta_{2}^{2}+1.8 \Delta_{3}\right) c_{1}+\left(0.75+2.1 \Delta_{2}\right) c_{2}+3 c_{3}- \\
-0.1 \Delta_{2} \Delta_{3}+0.25 \Delta_{3}+1.2 \Delta_{4}-\Delta_{5}
\end{gathered}
$$

(слагаемые, содержашие $\ln \theta$, сокрашаются при подстановке (53)). Из сказанного с учетом формул (34), (49) для матрицы $V$ вытекает

Tеорема 4. Пусть в формулах (48)

$$
r_{1}^{\prime}(y)=\nu c_{11}(y), \quad r_{2}=\text { const } \neq 0 .
$$

Тогда пара матрии и $=\left\|U_{i j}\right\|, \quad \mathrm{v}=\left\|V_{i j}\right\|$ с нулевым следом и әлементами (48), (50), построенныцми указанным выше образом, - решение системы (35). 
ЗАмечАниЕ 1. Аналогично могут быть построены решения системы (35) при $\operatorname{deg} w=g, \operatorname{deg} \Delta=2 g+1,2 g+2, g \in \mathbb{N}$. В случае нечетной $\operatorname{deg} \Delta$ матрица $\mathrm{u}(x, y)$ вследствие равенств $\mathrm{Sp} \mathrm{u}=0, \operatorname{det} \mathrm{u}=-\Delta_{0}=0$ подобна нильпотентной жордановой клетке второго порядка. Отсюда, в частности, вытекает, что в этом случае оператор $L=i \partial_{x}+\lambda \mathrm{u}$ не входит (с точностью до калибровочной эквивалентности) в класс конечнозонных операторов задачи $N$ волн, изученный в [7]. Можно доказать, что в случае четной $\operatorname{deg} \Delta\left(\Delta_{0} \neq 0\right)$ имеет место вложение в указанньй класс. Отметим, что для класса киральных полей Польмайера, построенного в [8] методом функций Бейкера-Ахиезера, имеет место (с учетом изоморфизма алгебр Ли $s o_{3}$ и $s l_{2}$ ) равенство $\operatorname{det} \mathrm{u}=1$.

Отметим также, что из построений пункта 4 следует, что функция $\alpha(x, y)=-i \ln (1+$ $w_{1}+w_{2}$ ) удовлетворяет уравнению sine-Gordon $\alpha_{x y}=4 \sin \alpha$. В самом деле, из (42) при $c_{11}=1$ и (44) с учетом (46), (47) получим

$$
v=e^{i \alpha}(\lambda-1)^{-1}, \quad u=\lambda-1+i \alpha_{x x} / 2-\alpha_{x}^{2} / 4
$$

Уравнение (1) для пары (5) с такими $u, v$ дает требуемое соотношение для $\alpha$.

ЗАмечание 2 . Полагая в (1) $U=i \lambda J+U_{0}(x, y)$, где $J=\operatorname{diag}(-1,1), U_{0}$ - матрица с нулевыми диагональными элементами, получим схему Захарова-Шабата (см. [2], c. 21). Так как в этом случае выполняются условия (3), то изложенный метод может быть применен, в частности, для построения алгебро-геометрических решений нелинейных уравнений, вкладывающихся в эту схему.

\section{Список литературы}

[1] Б. А. Дубровин, И. М. Кричевер, С. П. Новиков. В кн.: Современные проблемы математики. Фундаментальные направления. Т. 4. М.: ВИНИТИ, 1985. С. 179-284.

[2] M. Абловиц, X. Сигур. Солитоны и метод обратной задачи. М.: Мир, 1987.

[3] Б. А. Дубровин. УМН. 1981. Т. 36. Вып. 2. С. 11-80.

[4] Б. А. Дубровин, С. П. Новиков. ЖЭТФ. 1974. Т. 67. Вып. 6. С. 2131-2144.

[5] В. Е. Захаров, А. В. Михайлов. ЖЭТФ. 1978. Т. 74. Вып. 6. С. 1953-1974.

[6] Д. Мамфорд. Лекции о тэта-функциях. М.: Мир, 1988.

[7] Б. А. Дубровин. В кн.: Современные проблемы математики. Т. 23. М.: ВИНИТИ, 1983. C. 33-78.

[8] И. В. Чередник. В кн.: Итоги науки и техники. Алгебра. Геометрия. Топология. Т. 18. М.: Винити, 1981. С. 73-150.

Поступила в редакцию 15.I.1996 г.

\section{D.P. Novikov, R. K. Romanovski \\ ON A METHOD OF CONSTRUCTION ALGEBRO-GEOMETRICAL SOLUTIONS OF ZERO-CURVATURE EQUATIONS}

The construction is proposed, which reduces the calculation of algebro-geometrical solutions to the zero curvature equations for $2 \times 2$ matrices under special conditions valid some applications to the solution of the Jacobi inversion problem on a hyperelliptic Riemann surface and to the Riccati equation. The results obtained are applied to the principal chiral field system. 